\title{
An on-line program to calculate respiratory sinus arrhythmia amplitude
}

\author{
GUSTAVO A. REYES DEL PASO \\ University of Granada, Granada, Spain
}

\begin{abstract}
A variety of evidence shows that the amplitude of respiratory sinus arrhythmia (RSA)-the peak-to-peak variations in heart rate caused by respiration-is a valid and reliable index of parasympathetic cardiac control. An on-line program to calculate RSA amplitude on a breath-by-breath basis is described. The validity of the RSA amplitude values obtained through the computer program were tested in two studies. From the first one, the correlations between RSA amplitude and a set of cardiorespiratory variables, under both rest and auditory stimulation conditions, are reported. In the second study, the effect of atropine administration on the RSA amplitude, under both rest and mental load conditions, was examined. The results of both studies are congruent with predictions and suggest the usefulness of the software.
\end{abstract}

Respiratory sinus arrhythmia (RSA) is a phenomenon that consists of cyclical fluctuations in heart rate in close correspondence with respiratory phase: increases in heart rate during inspiration and decreases during expiration, although with certain delays or advances as a function of respiratory rate. These fluctuations depend on the respiratory pattern. Thus, the patterns characterized by low rates and high amplitudes increase the RSA amplitude to the maximum, whereas the patterns characterized by high rates and low amplitudes reduce it drastically (Eckberg, 1983).

RSA is the result of a complex interaction of various physiological mechanisms. These include mechanical effects related to the changes of blood pressure linked to respiratory activity, lung inflation receptors, chemoreceptors, baroreceptors, and, finally, the existence of a coupling mechanism at a central level between the respiratory and cardiovascular rhythms ("Breathing," 1978; Davidson, Golner, \& McCloskey, 1976; Davis, McCloskey, \& Potter, 1977; Eckberg, Kifle, \& Roberts, 1980; Koepchen, Klüssendorf, \& Sommer, 1981; Pinsky, Summer, Wise, Permutt, \& Bromberger-Barnea, 1983). Yet although RSA is the result of the interaction of these physiological processes, the final generators of RSA are cyclical vagal discharges due to the inhibition of vagal efferent activity during inspiration. The administration of atropine and vagotomy eliminate $R S A$, and variations in heart rate linked to breathing can be predicted on the basis of efferent cardiac vagal activity (Katona, Poitras, Barnett, \& Terry, 1970).

Part of this research was supported by a grant from the Commission of the European Communities Medical and Health Research Program ("Concerted Action: Quantification of Parameters for the Study of Breakdown in Human Adaptation"; project leader, Andrew Steptoe). Correspondence should be addressed to G. A. Reyes del Paso, Departamento de Personalidad, Evaluación y Tratamiento Psicológico, Facultad de Filosofía y Letras (Edificio B), University of Granada, 18011 Granada, Spain.
Given this vagal modulation of RSA, it has been proposed that its amplitude can be used as a noninvasive index of the level of the cardiac control mediated by the parasympathetic branch of the autonomic nervous system. In a series of important studies, Katona and colleagues (see Katona \& Jih, 1975) have examined this hypothesis in dogs by using invasive measures of the vagal influences on the heart and a variety of experimental stages, which included the increase (induced by a rise in blood pressure) as well as the decrease (induced by atropine administration) in the levels of the parasympathetic cardiac activity while the sympathetic control was partially or completely blocked. RSA was found to be an accurate indicator of vagal cardiac control under all conditions. The correlation obtained between both variables was $.969 \pm .024$. This result validates the use of RSA as a noninvasive index of parasympathetic cardiac control, explaining over $90 \%$ of the variance in the RSA for vagal influences. These results have been replicated in several studies with other animals. The correlations found oscillate between 0.83 and 0.97 (Fouad, Tarazi, Ferrario, Fighaly, \& Alicandro, 1984; Lipson \& Katona, 1979; Raczkowska, Eckberg, \& Ebert, 1983).

Given the aforementioned experimental results, a program to analyze RSA amplitude on a breath-by-breath basis has been developed. The program was designed with enough processing power to calculate RSA amplitude online from the simultaneous recordings of respiration and beat-by-beat heart rate.

\section{Description}

The program software is written in GW-BASIC and has been developed from computer programs designed previously for the analysis of respiratory activity and RSA. The method used involves simultaneous signal acquisition, analysis, and graphic display in real time. At the same time as the data from the respiratory activity and the heart rate are being acquired, the program analyzes the respiratory activity by obtaining the initial and final points of 
the inspiratory and expiratory phases of each breathing cycle. Once this is done, the minimum heart period from the sample that signals the beginning of the inspiration to the sample that signals the end of the inspiration (when the vagal activity is inhibited) and the maximum heart period from the sample that signals the beginning of the expiration until the time indicated by the sample that signals the beginning of the inspiration in the following respiratory cycle (period in which the vagal activity is present) are obtained. RSA amplitude is defined as the difference in milliseconds between the maximum and the minimum heart periods found under these conditions. When the minimum heart period during inspiration is longer than the maximum heart period during expiration, an RSA amplitude value of zero is assigned to that respiratory cycle. However, before this final analysis, and because of the existence of changes in the temporary phase relationships of the cardiac activity with respect to the respiratory activity as a function of the respiratory rate, the program analyzes each respiratory period and imposes certain delays or advances in the above respiratory points in terms of its duration. This adjustment is carried out in accordance with the results obtained by Eckberg (1983) with respect to the average timing of onset of heart period shortening and lengthening within the respiratory cycle as a function of the respiratory period. The result of the analysis is displayed on the screen of a computer by a vertical line about $1 \mathrm{~cm}$ wide, which increases or decreases in length according to the value of RSA amplitude in each respiratory cycle. This line is inserted within an $x, y$ plot and located exactly at the center on the horizontal axis, the vertical axis being scaled from 0 to 10 . For each experimental trial or session, the program obtains, displays on screen, and stores to disk the average RSA amplitude, the variability of the values of RSA amplitude (successive difference mean square), the average heart rate, the average breathing amplitude, the minute ventilation volume, the respiratory rate, and the average inspiratory, expiratory, and respiratory periods. The breathing amplitude is defined as the difference in analog-to-digital conversion values between the samples that signal the starting and finishing points of inspiration; the minute ventilation volume is defined as the sum of the breathing amplitudes within each analysis time expressed in terms of amplitude per minute; the respiratory rate is defined as the number of respiratory cycles within each analysis time expressed in terms of cycles per minute; the inspiratory period is defined as the time in seconds between the samples that indicate the starting and finishing points of the inspiration; the expiratory period is defined as the time in seconds between the samples that indicate the finishing point of inspiration-starting point of expiration and the finishing point of expiration; and the respiratory period is defined as the time in seconds between samples that indicate the starting points of inspiration in consecutive respiratory cycles. The program also stores to disk the individual values of RSA amplitude and the respiratory parameters in each breathing cycle, and it provides the option of storing the direct physiological samples.

\section{Hardware Requirements}

The program has been designed for a 12-bit analog-todigital converter, Model ANL-947, from MED Associates. However, the program can easily be adapted to other types of converters. It runs on the IBM PC or AT and compatibles, under MS-DOS. Given that the processing of the physiological variables is carried out at the same time as they are being acquired, it is necessary to obtain a good physiological recording before using the program, taking care to avoid artifacts, especially in the recording of heart rate. A high-frequency filter for the respiratory activity recording is recommended, set at $3-\mathrm{Hz}$ cutting frequency, to eliminate fast fluctuations superimposed on the respiratory signal. So that one may take advantage of the color contrasts available for displaying the results, the use of a color monitor is recommended.

\section{Validation and Accuracy Studies}

The validity of the the software's RSA amplitude values as indices of cardiac parasympathetic control has been tested according to two strategies, one correlational (examination of the relationship between the RSA amplitude and various cardiorespiratory variables) and the other experimental (pharmacologically blocking the vagal cardiac activity). The accuracy of the RSA amplitude values obtained with the program has been tested through comparison with careful hand scoring, in which the same adjustment of the phase time relationship between cardiac and respiratory activity was used.

Correlational study. Forty-two psychology students between 18 and 23 years of age took part in a study in which the relationship between the RSA amplitude and various cardiorespiratory variables were evaluated (breathing rate and amplitude, heart rate, heart rate variability, etc.) under both rest conditions and stimulation. An auditory stimulus-distorted sound of $400 \mathrm{~Hz}, 100 \mathrm{~dB}, 0.5-\mathrm{sec} \mathrm{du}$ ration, and instantaneous rise time-was presented after $10 \mathrm{~min}$ of rest. Two 120 -sec periods from the rest condition (Minutes 5-6 and 8-9) and the $80 \mathrm{sec}$ following stimulus presentation were selected for analysis. As can be seen in Table 1, the correlations obtained are totally congruent with the physiological literature on the topic. The RSA amplitude increases with decreases in the respiratory rate and increases in the breathing amplitude. On the other hand, when the RSA amplitude increases, the heart period and heart-period variability increase too. These correlations were observed under both rest and stimulation conditions.

Blocking study. Six medical students between 23 and 25 years of age participated in a study in which the RSA amplitude was assessed under rest and mental load conditions both before and after the cardiac vagal activity was blocked by atropine administration. A memory search and counting task developed by Massaro (1975) was used for the mental load condition. The subjects had to memorize four letters presented on a computer screen (memory set). Then, single letters appeared on the screen at 3-sec intervals, the subject having to decide whether or not the letter belonged to the memory set. If the decision was affir- 
Table 1

Correlation Between RSA Amplitude and Cardiorespiratory Variables, in Both Rest and Stimulation Periods

\begin{tabular}{lcc}
\hline & Rest & Stimulation \\
\hline Respiratory rate & -.643 & -.618 \\
Respiratory period & .684 & .513 \\
Expiratory period & .725 & .616 \\
Inspiratory period & .506 & .553 \\
Respiratory amplitude* & & .561 \\
Heart period & .449 & .568 \\
Heart period standard deviation & .613 & .495 \\
Heart period successive difference mean square & .809 & .746 \\
Heart rate & -.467 & -.557 \\
Heart rate standard deviation & .578 & .328 \\
Heart rate successive difference mean square & .804 & .540 \\
\hline
\end{tabular}

*The transducer used for the recording of the respiratory activity did not provide absolute values of amplitude. For the stimulation period, the percentage change in the $80 \mathrm{sec}$ prior to the presentation of the stimulus with respect to the 15 previous seconds were obtained for the respiratory amplitude.

mative, the subject had to press a button with the right hand. At the same time, the subject had to count mentally the number of times each letter of the memory set appeared on the screen. The structure of the experimental session was the following: 10 min of rest in which the last $200 \mathrm{sec}$ were taken as the baseline for the 'analysis, followed by the memory search task, which lasted $5 \mathrm{~min}$ and during which the recording of the initial $200 \mathrm{sec}$ was obtained for analysis. Once this first stage was completed, the drug was administered intravenously with a small catheter previously installed. The drug was atropine sulphate, administered at a dose of $.03 \mathrm{mg} / \mathrm{kg}$ or less until the heart rate was stabilized. Then the same procedure as that for the first stage was repeated.

The results show that RSA amplitude was greatly reduced after drug administration, the reduction being statistically significant both during baseline [97.7-6.5 msec; $F(1,5)=$ $30.82, p<.0001]$ and during memory search [55$4.6 \mathrm{msec} ; F(1,5)=177.2, p<.0001]$. The significance of the reduction in RSA amplitude during the second task in comparison with its baseline after atropine administration $[F(1,5)=13.19, p<.05]$ shows the persistence of a certain vagal activity after the blockade, as well as the sensitivity of this software to assess parasympathetic cardiac control.

In this study, the values obtained with the program and with careful hand scoring were compared, according to the same method. The results obtained through hand scoring were similar to those obtained with the program (98.6 msec during first baseline, $54.6 \mathrm{msec}$ during first task, $6.8 \mathrm{msec}$ during second baseline, and $4.9 \mathrm{msec}$ during the second task).

\section{Program Availability}

This software can be obtained by RN electronic mail (castellar@ugr.es) or from G. A. Reyes del Paso, Departamento de Personalidad, Evaluación y Tratamiento Psicológico, Facultad de Filosofía y Letras (Edificio B), Universidad de Granada, 18011 Granada, Spain.

\section{REFERENCES}

Breathing and control of heart rate (1978). British Medical Journal, 2, 1663-1664. (Editorial)

DAvidson, N. S., Golner, S., * MCCloskey, D. I. (1976). Respiratory modulation of baroreceptor and chemoreceptor reflexes affecting heart rate and cardiac vagal efferent nerve activity. Journal of Physiology, 259, 523-530.

Davis, A. L., McCloskey, D. I., Potter, E. K. (1977). Respiratory modulation of baroreceptor and chemoreceptor reflexes affecting heart rate through the sympathetic nervous system. Journal of Physiology, 272, 691-703.

ECKBERG, D. L. (1983). Human sinus arrhythmia as an index of vagal cardiac outflow. Journal of Applied Physiology, 54, 961-966.

Eckberg, D. L., Kifle, Y. T., \& Roberts, V. L. (1980). Phase relationship between normal human respiration and baroreflex responsiveness. Journal of Physiology, 304, 489-502.

Fouad, F. M., Tarazi, R. C., Ferrario, C. M., Fighaly, S., \& ALCANDro, C. (1984). Assessment of parasympathetic control of the heart by a noninvasive method. American Joumal of Physiology, 246, H838-H842.

KAtonA, P. G., Jih, R. (1975). Respiratory sinus arrhythmia: A noninvasive measure of parasympathetic cardiac control. Journal of Applied Physiology, 39, 801-805.

Katona, P. G., Poitras, J. W., Barnett, G. O., Terry, B. S. (1970). Cardiac vagal efferent activity and heart period in the carotid sinus reflex. American Journal of Physiology, 218, 1030-1037.

Koepchen, H. P., Klüssendorf, D., \& Sommer, D. (1981). Neurophysiological background of central neural cardiovascular-respiratory coordination: Basic remarks and experimental approach. Journal of the Autonomic Nervous System, 3, 335-368.

LiPson, D., KATONA, P. G. (1979). Respiratory sinus arrhythmia: A noninvasive assessment of parasympathetic chronotropic cardiac control in the conscious dog. Federation Proceedings, 38, 990.

MASSARO, D. W. (1975). Experimental psychology and information processing. Chicago: Rand McNally.

Pinsky, M. R., Summer, W. R., Wise, R. A., Permutt, S., \& BROMBERGER-BARNEA, B. (1983). Augmentation of cardiac function by elevation of intrathoracic pressure. Journal of Applied Physiology, 54, 950-955.

RACZKowsKa, M., ECKBerg, D. L., E EeRT, T. J. (1983). Muscarinic cholinergic receptors modulate vagal cardiac responses in man. Joumal of the Autonomic Nervous System, 7, 271-278.

(Manuscript received December 5, 1990; revision accepted for publication October 17, 1991.) 